\title{
Sensitivity Analysis of Models for High Consistency Refining Process
}

\author{
Patrick Höhn*1 and Wolfgang Birk ${ }^{1}$
}

\begin{abstract}
This paper deals with the sensitivity analysis of models for the high consistency refining process in the pulp and paper industry. Several models reported in literature are considered, which are screened for their potential use in the design of controllers for refiners or in the optimization of refiner operation. For the two most promising models a sensitivity analysis is conducted which suggests that the temperature profile inside the refiner and the inlet consistency are the most important process variables. It is further suggested that both variables variables are utilized to control the refining process based on these models. Moreover, computer simulations with constant, linear increasing and parabolic temperature profiles are performed, which confirm the importance of the temperature profile for the entropy based model.
\end{abstract}

\section{INTRODUCTION}

The Swedish pulp and paper industry has 12 percent share of the Swedish exports. Moreover, it also accounts for half of the energy consumption of the whole Swedish Industry [16]. Therefore, in order to stay competitive and minimize costs the energy efficiency of the whole paper production process needs to be continuously optimized.

The whole manufacturing process splits in two distinct consecutive sections, the pulp mill and the paper machine where the pulp is used to produce the final paper product. The pulp mill itself consists of process steps for debarking, chipping, chemical or mechanical pulping, refining, chemical treatment and screening. During chemical pulping the wood chips from previous process steps are treated to dissolve ligning surrounding the fibers. Mechanical pulping avoids the cooking procedure and creates the pulp by mechanically breaking the wood in a thermo-mechanical pulp (TMP) refiner. Secondary refiners are comparable to refiners used in chemical pulping processes.

The refining process involves a device similar to a centrifugal pump consisting of two discs - one rotating and one fixed - with bars and grooves to accomplish a grinding effect. Inside the gap between the two plates fibers are treated to reach desired properties for the final paper production (see Fig. 1) which accounts for approximately 30 percent of the total energy consumption. Huhtanen et. al addressed this by designing the refiner according to the theory of rotational pumps [4].

More options for cost reductions were evaluated in [14]. It was shown that both improvements in the energy efficiency and variations of quality parameters can yield large economic benefits. The latter also increases the reliability of the paper

*Corresponding author:patrick.hohn@ltu.se

${ }^{1}$ Control Engineering Group, Department of Computer Science, Electrical and Space Engineering, Luleå University of Technology, SE-971 87 Luleå, Sweden



Fig. 1. Schematic sketch of a refiner with arrows indicating the direction of primary flow

manufacturing process. Considering that around 35 percent of all control loops in pulp and paper factories are not sufficiently tuned [12], control schemes which do not depend on manual tuning or require little user interaction could be a major contribution to the above goals. Karlström et. al proposed a model predictive controller for refiners used in thermo-mechanical pulp (TMP) processes [6]. However, an actual implementation requires a deeper understanding of models for the refining process. These provide already the relationships of a large number of model parameters and input variables involved in the refining process.

The aim of this paper is to reveal which of those parameters or variables have a large impact on the behavior of the refiner. This causes large implications on the implementation of models. Firstly, model parameters with a high sensitivity need to be determined with high accuracy. Secondly, input variables which largely affect the process are good candidates for control inputs and are of great importance for the modeling of the refining process.

Using a sensitivity analysis of the available models will yield indications on the impact of various parameters and variables. Thus, the contribution of this paper is to determine the most promising control variables for new control designs based on the analyzed models.

The layout of the paper is as follows. The considered models comprising of two speed and consistency, one enthalpy and one entropy based models, are described in the next section. 
Section III presents the large dependency of the output variables on the temperature in the refining zone. Thereafter the utilized models are further analyzed in a simulation study (Section IV). The paper closes with conclusion and an outlook on future work (Section V).

\section{REVIEW AND SCREENING OF EXISTING MODELS}

Many refiner models were developed throughout the years. Four of these which can be used to improve the understanding and energy consumption of the refining process are reviewed and screened for use in advanced control schemes. The first modeling approach is related to the average velocities inside refiners and was already proposed in 1979 [15]. It gives insights in the estimation of the viscosity which is an important flow parameter of the pulp. The second model introduces an approach to estimate the consistency profile inside the refiner with respect to the applied energy. Entropy generation during the refining process is the basis for the third model which gives very detailed information on the mass flows. The last modeling approach treats the refiner as a black box and relates the applied work to the outgoing steam flow rate.

\section{A. Flow speed model}

The Flow speed model, as presented in [15], uses a simplistic model approach assuming that the grooves in the fixed and rotating refiner disc are facing each other. Under condition of laminar flows and no secondary flows the profile of the average velocities is determined. Model inputs are the dimensions of the flow channel $a$ and $b$, the pressure gradient $\frac{d p}{d r}$ and the absolute viscosity $\mu$. All model inputs, except the viscosity, can be measured directly with commercially available sensors [2]. Fox overcame this limitation by estimating the viscosity by curve fitting velocities which were measured by an optical sensor in an experimental refiner made of Plexiglas.

As the setup of the flow speed model cannot be replicated on mill scale refining processes, the velocities cannot be obtained in the same way and thus, the model cannot be utilized for advanced control schemes.

\section{B. Consistency model}

The modeling approach for the consistency by [8] assumes that the latent heat of the steam $L$ is independent of the pressure inside the refiner, i. e. constant. Moreover, the applied energy is considered to be uniformly distributed over the radius. This leads to an expression for the consistency profile:

$$
C(r)=\frac{L \cdot C\left(r_{1}\right)}{L-C\left(r_{1}\right) \cdot S E \cdot \frac{r^{2}-r_{1}^{2}}{r_{2}^{2}-r_{1}^{2}}}
$$

where $C(r)$ denotes the consistency as a function of radius, $r_{1}$ and $r_{2}$ the inner and outer radii of the refiner disc, $L$ the latent heat of the steam and $S E$ the specific energy applied to the pulp. This model was only validated in the special case when the feeding is breaking down due to a too high inlet consistency. However, it is also assumed to be applicable for other operating conditions.

As the Consistency model relates the measurable inlet consistency $C\left(r_{1}\right)$ with the commonly used control variable specific energy $S E$, it is considered suitable for further analysis.

\section{Entropy model}

The entropy model, as presented in [7], is based on several assumptions:

- the temperature profile inside the refiner is known

- the flow profile is laminar in rotational direction

- the pulp is fluidized and following Stoke's theorem on viscosity

- there exist a perfect mixing and adiabatic conditions due to the complex flows inside of the refiner

- the steam and water inside the refiner can be treated as being in the saturated state

- the generated entropy in an infinitesimal control volume can be determined by the expression for the viscous dissipation

- inside the refining zone the water is bound to the fibers

- the steam phase is driven by a pressure gradient and follows Darcy's law for porous flows.

- the hydraulic permeability is a function of the volume fraction of the pulp.

- the PDEs which are describing the refining from a macroscopic perspective can be simplified to steadystate spatially dependent differential equations.

The model uses a temperature profile $T(r)$, input consistency $C\left(r_{1}\right)$, applied energy $S E$, gap clearance $b$ and pulp mass flow rate $\dot{m}_{P}$ as inputs and generates the consistency $C(r)$, energy consumption $W(r)$ and phase mass flow profile $\dot{m}_{i}(r)$. Engberg showed in [1] that the model gives a good representation of reality. At first, the mass flow rates of the liquid $\dot{m}_{w o}$ and fiber fraction $\dot{m}_{p}$ are determined using the total mass flow rate $\dot{m}_{t}$ and the inflow consistency $C\left(r_{1}\right)$ :

$$
\begin{aligned}
\dot{m}_{p} & =C\left(r_{1}\right) \cdot \dot{m}_{t} \\
\dot{m}_{w o}\left(r_{1}\right) & =\dot{m}_{t}-\dot{m}_{p}
\end{aligned}
$$

The total energy input to the refiner $W_{\text {tot }_{o}}$ can be determined from the definition of the specific energy $S E$ by:

$$
W_{\text {tot }_{o}}=S E \cdot \dot{m}_{p}
$$

In order to derive the energy consumption over the radius the total viscosity $\mu$ is derived so that the total work over the entire plate equals the measured work applied to the refiner.

$$
\mu=\frac{2 \cdot b \cdot W_{\text {tot }_{o}}}{\pi \cdot \omega^{2} \cdot\left(r_{2}^{4}-r_{1}^{4}\right)}
$$

Then, the total energy consumption between the inner radius and the discrete radii $w_{t o t_{o}}$ can be calculated to be:

$$
w_{\text {tot }_{o}}(r)=\frac{\mu \cdot(r \cdot \omega)^{2}}{b}
$$


As the most important part of the whole model, the generated entropy in the different radial sections $S_{g e n}$ results in:

$$
S_{g e n}(r)=\int_{r_{1}}^{r} 2 \cdot \pi \cdot C\left(r_{1}\right) \cdot \rho \cdot \frac{w_{\text {toto }_{o}}}{T+273 K} d \rho
$$

Utilizing entropies determined from steam tables using the temperature profile and the previous equations, results in the mass flow rates for water $\dot{m}_{w o}$ and steam $\dot{m}_{s o}$ :

$$
\begin{aligned}
\dot{m}_{s o}(r)= & \frac{S_{g e n}(r)-\dot{m}_{w o}\left(r_{1}\right) \cdot\left(s_{2}(r)-s_{2}(r 1)\right)}{s_{1}(r)-s_{2}(r)} \\
& -\frac{\dot{m}_{p}(r) \cdot C_{P} \cdot \log \left(\frac{T(r)+273 K}{T\left(r_{1}\right)+273 K}\right)}{s_{1}(r)-s_{2}(r)} \\
\dot{m}_{w o}(r)= & \dot{m}_{w o}\left(r_{1}\right)-\dot{m}_{s o}(r)
\end{aligned}
$$

Thus, the consistency $C(r)$ can be determined to be:

$$
C(r)=\frac{\dot{m}_{p}}{\dot{m}_{p}+\dot{m}_{w o}(r)}
$$

Within the cited paper [7] measurements and estimates of the

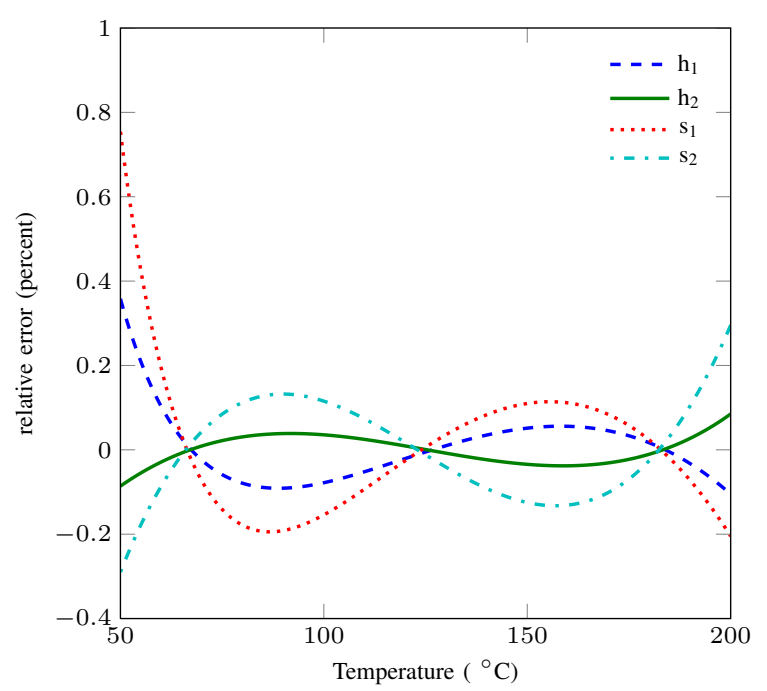

Fig. 2. Relative error of the fitted functions compared to the complete model by IAPWS

consistency were presented and it is stated that were fitting well.

As the entropy model gives large insights in the refining process and utilizes measurable variables, it is considered to be suitable for advanced control schemes and, thus, further analyzed.

\section{Enthalpy model}

The enthalpy model approach, as presented in [11], includes a smaller amount of assumptions compared to the previous model: (i) the heat loss in the motor is proportional to the motor load; (ii) no condensation of back flowing steam on the incoming wood chips occurs; (iii) the refiner is a black box just considering input-output behavior. As the $\mathrm{HC}$ process in this study is already assuming pre-made pulp, some of the equations from [11] are adjusted appropriately. Another difference is that the current analysis does not include any flows of dilution water. Therefore the equations simplify further. Based on these assumptions the steam flow rate at the outlet of the refiner is determined. It should also be noted that the model does not account any work for the fibrillation of the fibers throughout the refining process. The model was validated with real mill data, as stated in [11]. Since the enthalpy model is using variables which are not measurable, e. g. the enthalpy of the steam, the model is not considered for further investigations within this paper.

Based on the screening in this section the entropy and consistency modeling approach are found useful for improvements in the refiner control and, thus, analyzed more detailed for important process variables in the next section.

\section{SENSITIVITY ANALYSIS}

It was decided to omit the speed model since it provides no insights in the energy consumption. The second model is directly related to the specific energy. Changes of consistency can be only caused by evaporation which is closely related to the thermodynamical work applied inside the refiner. Thus, the influence of the different model inputs and parameters on the consistency profile is further analyzed. Similarly, the equation for the mass flow rate of steam is selected from the entropy model because the creation of steam is causing an energy consumption which is not contributing to the change of the fiber properties. The fourth model is omitted because it regards the refiner as a black box and calculates the outflow mass rate of steam from the refiner. Therefore, the model might miss the largest potential of energy savings inside the refiner.

As mentioned in the previous section, the entropy model requires values for the entropy and enthalpy. The values for the analysis within this study are taken from steam tables defined by the IAPWS IF-97 Standard [17]. The enthalpy equations from the cited standard require beside the temperature profile also the pressure profile. Moreover, precise enthalpy and entropy values are needed for the design of thermal turbomachines like turbines. This results in very complex equations in the cited standard. However, the high precision of the previous equations in this section is not required here. Thus, to perform a sensitivity analysis of (2) the equations for the entropy and enthalpy can be simplified by curve fitting. Fig. 2 shows the relative error of the fitting using the equations below:

$$
\begin{aligned}
h_{1} & =0.001023 \cdot T^{2}+4.02 \cdot T+6.516 \\
h_{2} & =-0.003798 \cdot T^{2}+2.319 \cdot T+2483 \\
s_{1} & =-1.138 \cdot 10^{-5} \cdot T^{2}+0.01363 \cdot T+0.05628 \\
s_{2} & =3.097 \cdot 10^{-5} \cdot T^{2}-0.01842 \cdot T+8.895
\end{aligned}
$$

Using this and the equations from Section II the partial differential functions for the mass flow rate of the steam can be determined, as given in (4) - (11).

The equations were evaluated numerically using the parameters from section IV for the case of a parabolic temperature 


$$
\begin{aligned}
& \frac{\partial \dot{m}_{s o}}{\partial T}=-\frac{1}{d} \cdot\left(\frac{C_{P} \cdot \dot{m}_{p}}{T+273.15}+\frac{C\left(r_{1}\right) \cdot d r \cdot \mu \cdot \omega^{2} \cdot \pi \cdot r^{4}}{2 \cdot b \cdot(T+273.15)^{2}}+\dot{m}_{w o}\left(r_{1}\right) \cdot\left(6.194 \cdot 10^{-5} \cdot T-0.01842\right)\right) \\
& -\frac{0.03205-8.47 \cdot 10^{-5} \cdot T}{d^{2}} \cdot\left(-C_{P} \cdot \dot{m}_{p} \cdot \log \left(\frac{T+273.15}{T\left(r_{1}\right)+273.15}\right)\right. \\
& \left.+\frac{C\left(r_{1}\right) \cdot d r \cdot \mu \cdot \omega^{2} \cdot \pi \cdot r^{4}}{2 \cdot b \cdot(T+273.15)}-\dot{m}_{w o}\left(r_{1}\right) \cdot\left(3.097 \cdot 10^{-5} \cdot T^{2}-0.01842 \cdot T-s_{2}\left(r_{1}\right)+8.895\right)\right) \\
& \frac{\partial \dot{m}_{s o}}{\partial C}=\frac{d r \cdot \mu \cdot \omega^{2} \cdot \pi \cdot r^{4}}{2 \cdot b \cdot(T+273.15) \cdot d} \\
& \frac{\partial \dot{m}_{s o}}{\partial d r}=\frac{C\left(r_{1}\right) \cdot \mu \cdot \omega^{2} \cdot \pi \cdot r^{4}}{2 \cdot b \cdot(T+273.15) \cdot d} \\
& \frac{\partial \dot{m}_{s o}}{\partial \mu}=\frac{C\left(r_{1}\right) \cdot d_{r} \cdot \omega^{2} \cdot \pi \cdot r^{4}}{2 \cdot b \cdot(T+273.15) \cdot d} \\
& \frac{\partial \dot{m}_{s o}}{\partial \omega}=\frac{C\left(r_{1}\right) \cdot d_{r} \cdot \mu \cdot \omega \cdot \pi \cdot r^{4}}{b \cdot(T+273.15) \cdot d} \\
& \frac{\partial \dot{m}_{s o}}{\partial \dot{m}_{w o}}=\frac{-3.097 \cdot 10^{-5} \cdot T^{2}+0.02154 \cdot T+s_{2}\left(r_{1}\right)-9.075}{d} \\
& \frac{\partial \dot{m}_{s o}}{\partial \dot{m}_{p}}=-\frac{C_{P}}{d} \cdot \log \left(\frac{T+273.15}{T\left(r_{1}\right)+273.15}\right) \\
& d=-4.235 \cdot 10^{-5} \cdot T^{2}+0.03205 \cdot T-8.83872 \\
& \frac{\partial C(r)}{\partial L}=\frac{C\left(r_{1}\right) \cdot\left(r_{2}^{2}-r_{1}^{2}\right)}{L \cdot\left(r_{2}^{2}-r_{1}^{2}\right)-C\left(r_{1}\right) \cdot S E \cdot\left(r^{2}-r_{1}^{2}\right)}-\frac{C\left(r_{1}\right) \cdot L \cdot\left(r_{2}^{2}-r_{1}^{2}\right)^{2}}{\left(L \cdot\left(r_{2}^{2}-r_{1}^{2}\right)-C\left(r_{1}\right) \cdot S E \cdot\left(r^{2}-r_{1}^{2}\right)\right)^{2}} \\
& \frac{\partial C(r)}{\partial C\left(r_{1}\right)}=\frac{L \cdot\left(r_{2}^{2}-r_{1}^{2}\right)}{L \cdot\left(r_{2}^{2}-r_{1}^{2}\right)-C\left(r_{1}\right) \cdot S E \cdot\left(r^{2}-r_{1}^{2}\right)}+\frac{\left(r_{2}^{2}-r_{1}^{2}\right) \cdot\left(r^{2}-r_{1}^{2}\right) \cdot C\left(r_{1}\right) \cdot L \cdot S E}{\left(L \cdot\left(r_{2}^{2}-r_{1}^{2}\right)-C\left(r_{1}\right) \cdot S E \cdot\left(r^{2}-r_{1}^{2}\right)\right)^{2}} \\
& \frac{\partial C(r)}{\partial S E}=\frac{\left(r_{2}^{2}-r_{1}^{2}\right) \cdot\left(r^{2}-r_{1}^{2}\right) \cdot C\left(r_{1}\right)^{2} \cdot L}{\left(L \cdot\left(r_{2}^{2}-r_{1}^{2}\right)-C\left(r_{1}\right) \cdot S E \cdot\left(r^{2}-r_{1}^{2}\right)\right)^{2}} \\
& \frac{\partial C(r)}{\partial r_{2}}=-\frac{2 \cdot\left(r^{2}-r_{1}^{2}\right) \cdot r_{2} \cdot C\left(r_{1}\right)^{2} \cdot L \cdot S E}{\left(L \cdot\left(r_{2}^{2}-r_{1}^{2}\right)-C\left(r_{1}\right) \cdot S E \cdot\left(r^{2}-r_{1}^{2}\right)\right)^{2}} \\
& \frac{\partial C(r)}{\partial r_{1}}=-\frac{C\left(r_{1}\right) \cdot L \cdot\left(2 \cdot r_{1} \cdot C\left(r_{1}\right) \cdot S E \cdot\left(r_{2}^{2}-r_{1}^{2}\right)-2 \cdot r_{1} \cdot\left(r^{2}-r_{1}^{2}\right) \cdot C\left(r_{1}\right) \cdot S E\right)}{\left(L \cdot\left(r_{2}^{2}-r_{1}^{2}\right)-\left(r^{2}-r_{1}^{2}\right) \cdot C\left(r_{1}\right) \cdot S E\right)^{2}}
\end{aligned}
$$



Fig. 3. Relative sensitivity from (4) - (10) normalized by the maximum value of the parameters, where temperature $T$ (solid), pulp mass flow rate $\dot{m}_{P}$ (loosely dotted), inflowing water mass flow rate $\dot{m}_{w o}\left(r_{1}\right)$ (dash dotdotted), angular velocity $\omega$ (loosely dash dotted), discrete radius $d r$ (dotted), inlet consistency $C\left(r_{1}\right)$ (dashed) and viscosity $\mu$ (dash dotted) profile. Results normalized by the maximum value of $\dot{m}_{s o}$ and the respective parameter or variable are shown in Fig. 3. Obviously the mass flow rate of the incoming water $\dot{m}_{w o}\left(r_{1}\right)$ is the most important variable which is very reasonable as there is no inflow of steam and thus, all steam must originate from the incoming water. However, as water is only added at the inlet area, the mass flow rate gives no information about the process inside the gap itself. It can also be clearly seen that the temperature $T$ is the second most important variable. Unlike the mass flow rate of water $\dot{m}_{w o}\left(r_{1}\right)$, it can be measured in different locations on the radius of the refiner. Thus, it is justified to consider the temperature as a key variable to describe the behavior inside of a refiner [3]. Unlike all other partial derivatives, the partial derivative with respect to the pulp flow rate $\dot{m}_{P}$ shows a clear curvature with a saturation region towards the outer radius $r_{2}$. This could arise from the fact that the fiber as sole solid fraction of the pulp affects the evaporation and condensation rates. As fourth least important partial differential the angular velocity of the rotor $\omega$ appears almost linear. The three least important contributions by the radial discretization $(d r)$, inlet consistency $(C)$ and viscosity $(\mu)$ seem to coincide with each other in an almost linear curve.

Unlike the entropy model, the consistency model does not require any simplifications for further analysis. The resulting 


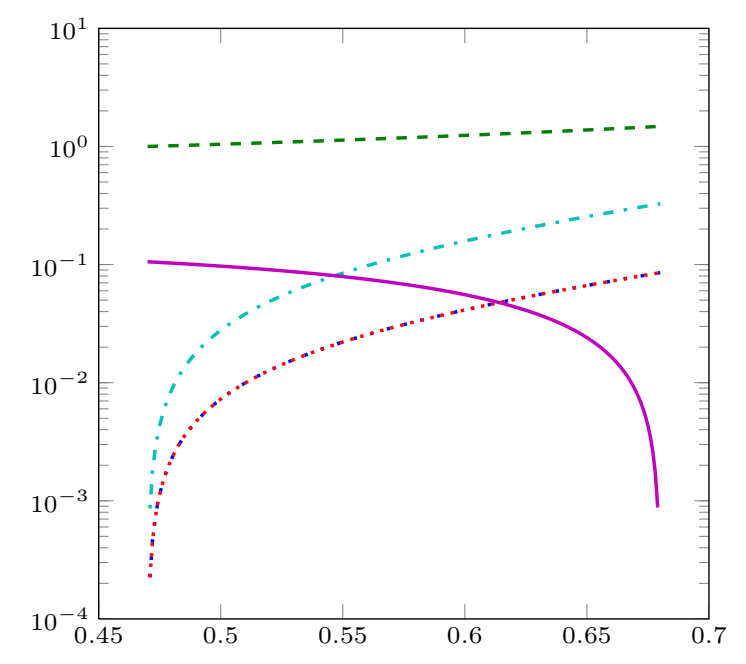

Fig. 4. Relative sensitivity from (12) - (16) normalized by the maximum value of the parameters, where inner radius $r_{1}$ (solid), outer radius $r_{2}$ (dash dotted), latent heat of steam $L$ (loosely dotted), specific energy $S E$ (dotted) and inlet consistency $C\left(r_{1}\right)$ (dashed)

partial derivatives are shown in (12) - (16) and plotted in Fig. 4. The graphs were normalized in the same way as in the entropy model. It is obvious that the inflow consistency has the largest influence on the resulting consistency profile. Thus, it is important to measure the inflow consistency precisely to obtain a good estimation of the whole profile. The second and third most important parameters are the inner and outer disc radius. Their influence was found to be highly dependent on the radial position. It should be also noted that the influence of the energy related terms in the equation is the smallest. The results of this section will be evaluated further by a simulation study in the next section.

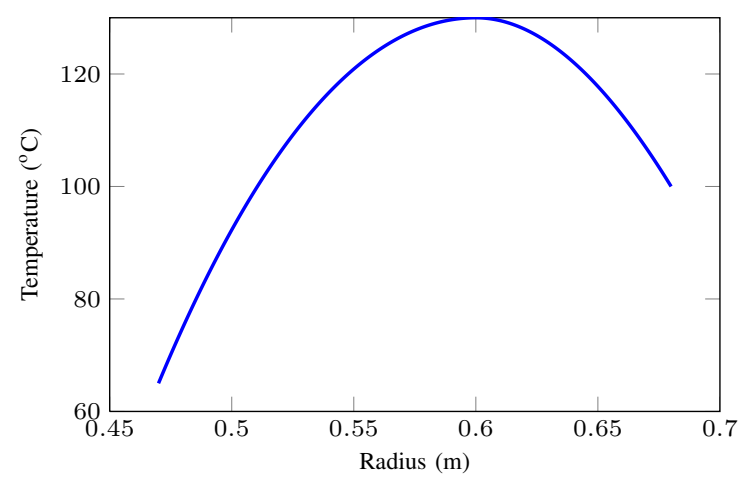

Fig. 5. Temperature profile used in the simulation study

\section{SIMULATION STUDY AND DISCUSSION}

This section is divided into three parts. As shown in the previous section the temperature profile has a large influence on the output of the entropy model. Thus, the first subsection discusses the temperature profiles further. These are then applied in the simulation study in the second part. Followed by this the results are discussed in the last part.

\section{A. Temperature profiles}

As the temperature was identified in the last section as a important input variable, temperature profiles are discussed in the next subsection. The temperature profile inside a refiner is regarded as a function over the radius with a distinct maximum in between the inner radius $r_{1}$ and outer radius $r_{2}$ [7]. Here three kinds of temperature distributions are considered, constant, linear and with a clear curvature. The latter is assumed to follow a cubic spline interpolation of the radius with three vertices. First vertex is the inner radius and the starting temperature in accordance with the temperature profile. Second vertex is the peak temperature and with its position at radius $0.6 \mathrm{~m}$. The choice of the position for the peak temperature was based on information from industrial partners. The third vertex is at the outer radius with the final temperature level.

There exist several approaches to alter the shape of the parabolic profile. The position of the peak can be affected by adjusting the inlet pressure [13]. Moreover, the peak value itself can be influenced by modifications in the plate pattern or by introducing evacuated segments [5] or by changes in pulp mass flow rate or hydraulic closing pressure [9].

\section{B. Simulation study}

The simulations in this study used different assumptions for the temperature profile inside the refiner, including a constant profile at a temperature of $100^{\circ} \mathrm{C}$, a linear rising profile between $65^{\circ} \mathrm{C}$ and $100^{\circ} \mathrm{C}$ and a parabolic one for the validation of the temperature influence (see Fig. 5). The specific heat of the fibers has been chosen to $0.4 \mathrm{~kJ} / \mathrm{kgK}$, a value that holds for pine wood [10]. All simulations assume a single disc refiner with an inner disc radius of $0.47 \mathrm{~m}$ and outer disc radius of $0.68 \mathrm{~m}$. The inflow temperature and consistency are $65^{\circ} \mathrm{C}$ and $32.43 \%$. It is known that the plate gap is reducing towards the outer radius, but for sake of simplicity, the plate distance has been assumed to be independent of the radius, i. e. $b(r) \equiv B$ which gives $\dot{W}_{\text {tot }}=\mu \pi \omega^{2}\left(r_{2}^{4}-r_{1}^{4}\right) / 2 B$. Most energy is consumed in the periphery of the segment. The simulation is only performed over this narrow band. The plate gap $b$ is set to $0.6 \mathrm{~mm}$ as initial value. The deviation measurement of the rotor position can be used as a variation around the initial value. Clearly, there can be both an offset and dynamics of the plate gap which remain unknown for the time being. For this paper the deviations are not considered. The specific energy is selected to be $1096 \mathrm{~kJ} / \mathrm{kg}$ and the mass flow rate $1.94 \mathrm{~kg} / \mathrm{s}$.

Using all parameters and variables above Fig. 6 - Fig. 9 were generated. They will be discussed in the next subsection.

\section{Discussion}

Fig. 6 shows the mass flow rates for the case of a constant temperature. In this case while the water is flowing through the refiner, steam is created and flowing in the same direction as the pulp and water. However, in the case of a linear rising temperature the steam starts to flow backward (see Fig. 7). This could be caused by an increasing pressure resulting 


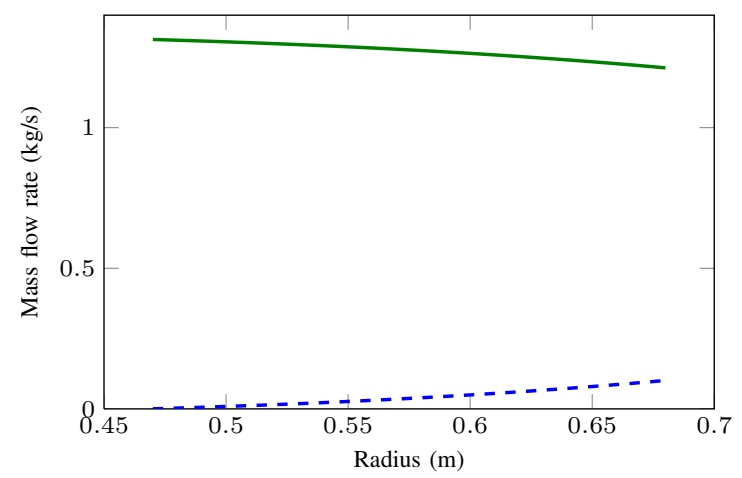

Fig. 6. Simulated mass flow rate results for a constant temperature profile,where mass flow rate of steam (dashed) and mass flow rate of water(solid)

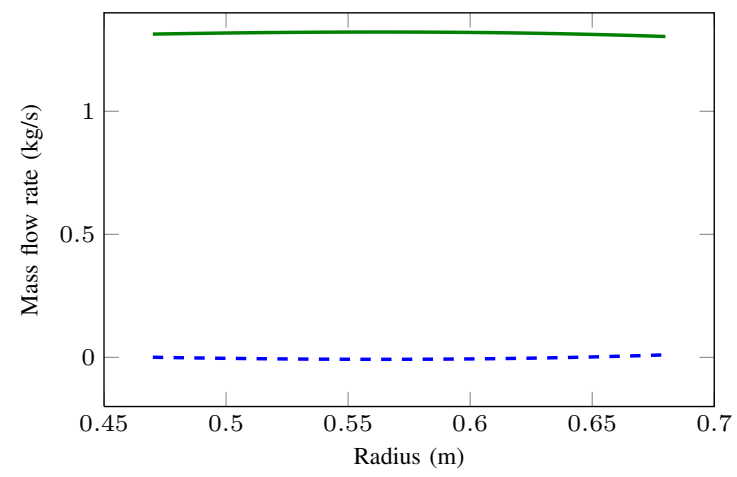

Fig. 7. Simulated mass flow rate results for a linear rising temperature profile,where mass flow rate of steam (dashed) and mass flow rate of water(solid)

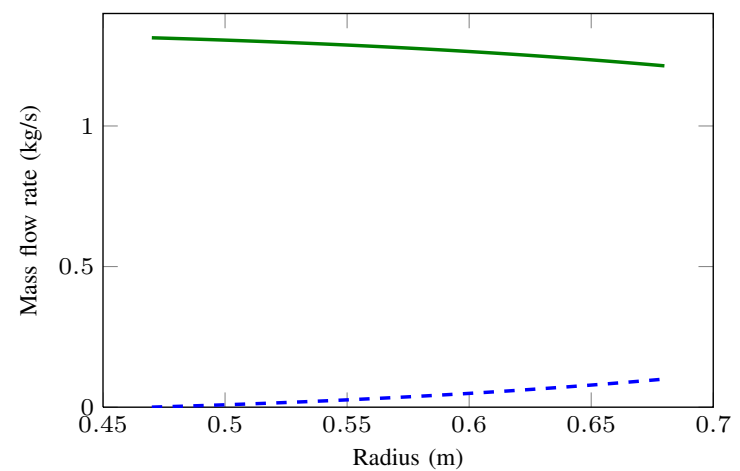

Fig. 8. Simulated mass flow rate results for a parabolic temperature profile given in Fig. 5, where mass flow rate of steam (dashed) and mass flow rate of water(solid)

from increasing evaporation as the temperature is rising. Due to the conservation of mass the forward mass flow rate of the water also increases unlike the drop in the constant temperature case. In the case of the parabolic temperature profile, as shown in figure 8 , the distribution throughout the radius changes significantly. Unlike in the cases before the profile shows a clear curvature. This also fits to the result from section III where the temperature affects the output over the whole radius range.

The consistency profile in the case of a constant temperature

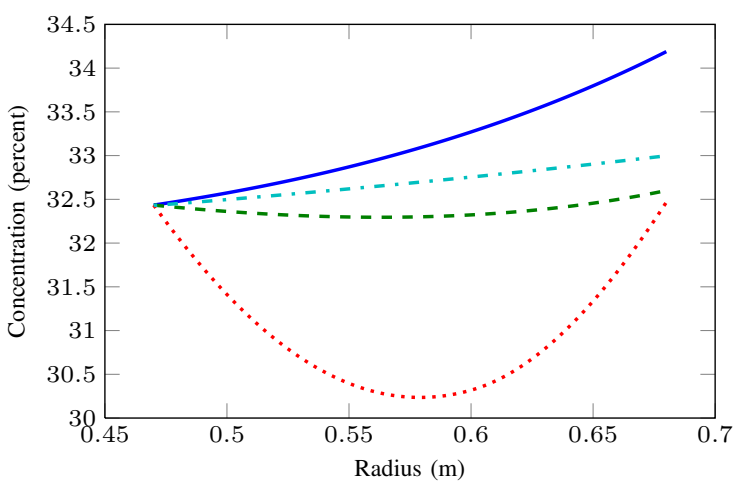

Fig. 9. Simulated concentration results for the entropy model where a constant temperature profile (solid), a linear rising temperature profile (dashed) and a parabolic temperature profile (dotted) is used and for the concentration model (dash dotted)

profile, as depicted in Fig. 9, fits to the expectations. As more steam is created from the inner radius to the outer radius the consistency should increase. In case of linear rising temperatures (see Fig. 9) the modeling approach by Miles and May shows an increase of consistency as expected. However, the modeling approach by Eriksson shows a decrease in consistency due to back flowing steam. Finally, in the case of the parabolic temperature profile, as shown in Fig. 9 the profile generated by the consistency model shows a similar shape like in the case of a linear rising temperature. This is not surprising as the consistency is very weakly dependent on the temperature just by small variations in the latent heat of the steam. Unlike this, the temperature profile is clearly reflected in the resulting consistency. The parabolic shape indicates that there exists an equilibrium point from which the steam is flowing in forward and backward direction.

\section{CONCLUSIONS AND OUTLOOK}

An outline of currently available models for refiners in the pulp and paper industry was given. Based on this a screening of the models for being suitable in more model based control schemes was performed. It was found that the entropy and the consistency modeling approach are most promising for this purpose.

The entropy model was further analyzed regarding its sensitivity with respect to its input variables where the mass flow rate of the inflowing water and the temperature profile inside the refiner were found to have the largest influence. This was also confirmed in the simulation study resulting in mass flow rates for water and steam and the consistency profiles. Thus, when considering the entropy model for a model predictive controller, it is well justified to use the temperature profile or the mass flow rate for water as control variable. For the consistency profile, it was shown that the modeling approach by Miles and May is completely insensitive to the temperature distribution. This is due to the assumption that the latent heat of the steam is considered to be independent of the pressure inside the refiner. Considering saturated steam inside the refiner, this also implies that the influence of the temperature is also neglected and is a clear weakness of the 
model. Thus, in this case for a control of the consistency profile the inlet consistency would be the most preferable choice.

Further analysis could consider uncertainties and measurement errors in the input data. Based on the results of this paper a system identification for the most important variables will be performed in order to design and implement a model predictive controller.

\begin{tabular}{|c|c|c|}
\hline \multicolumn{3}{|c|}{ APPENDIX } \\
\hline Symbol & Explanation & Unit \\
\hline$\mu$ & viscosity of the pulp & $\mathrm{N} /\left(\mathrm{s}^{2} \mathrm{~m}^{2}\right)$ \\
\hline$r$ & radius & $\mathrm{m}$ \\
\hline$C$ & consistency of pulp & \\
\hline$r_{1}$ & inner radius of refiner disc & $\mathrm{m}$ \\
\hline$r_{2}$ & outer radius of refiner disc & $\mathrm{m}$ \\
\hline$s_{1}$ & local entropy of the steam & $\mathrm{J} /(\mathrm{kg} \mathrm{K})$ \\
\hline$S E$ & specific energy applied in refiner & $\mathrm{kWh} / \mathrm{t}$ \\
\hline$b$ & gap clearance & $\mathrm{mm}$ \\
\hline$\omega$ & Angular velocity of rotor & $\mathrm{rad} / \mathrm{s}$ \\
\hline$C_{P}$ & specific heat capacity of fibers & $\mathrm{kJ} /(\mathrm{kgK})$ \\
\hline$C_{W}$ & specific heat capacity of water & $\mathrm{kJ} /(\mathrm{kgK})$ \\
\hline$L$ & latent heat of the steam & $\mathrm{kJ} / \mathrm{kg}$ \\
\hline$\dot{m}_{t}$ & $\begin{array}{l}\text { total mass flow rate through the } \\
\text { refiner }\end{array}$ & $\mathrm{kg} / \mathrm{s}$ \\
\hline$\dot{m}_{s o}$ & $\begin{array}{l}\text { local steam mass flow rate through } \\
\text { the refiner }\end{array}$ & $\mathrm{kg} / \mathrm{s}$ \\
\hline$\dot{m}_{w o}$ & $\begin{array}{l}\text { local water mass flow rate through } \\
\text { the refiner }\end{array}$ & $\mathrm{kg} / \mathrm{s}$ \\
\hline$W_{\text {tot }_{o}}$ & total energy applied to refiner & $\mathrm{kW}$ \\
\hline$w_{\text {toto }_{o}}$ & $\begin{array}{l}\text { energy applied between inner and } \\
\text { local radius }\end{array}$ & \\
\hline$S_{g e n}$ & $\begin{array}{l}\text { entropy generated between inner } \\
\text { and local radius }\end{array}$ & $\mathrm{J} /(\mathrm{sK})$ \\
\hline$s_{2}$ & local entropy of the water & $\mathrm{J} /(\mathrm{kg} \mathrm{K})$ \\
\hline$\dot{m}_{P}$ & $\begin{array}{l}\text { pulp(fiber) mass flow rate through } \\
\text { the refiner }\end{array}$ & $\mathrm{kg} / \mathrm{s}$ \\
\hline
\end{tabular}

\section{ACKNOWLEDGMENT}

The support by the SCOPE consortium and Mondi Dynäs $\mathrm{AB}$ is warmly appreciated. Funding provided by the European Structural Funds is hereby gratefully acknowledged.

\section{REFERENCES}

[1] B. Engberg and J.-E. Berg, "A comparative study of models describing high consistency refining," in Proceedings of International Mechanical Pulping Conference. Mid Sweden University, Department of Natural Sciences, Engineering and Mathematics, 2011, p. 96-100.

[2] O. Eriksen and L. Å. Hammar, "Literature survey. measurement techniques suitable for the refining zone of disc and conical LC refiners," Technical report STFI-Packforsk, Tech. Rep., 2005. (Available Online)

[3] K. Eriksson and A. Karlström, "Refining zone temperature control: A good choice for pulp quality control?" in Proceedings - 2009 International Mechanical Pulping Conference, IMPC 2009, 2009, pp. $67-74$.

[4] J. P. Huhtanen, "Theoretical background of new energy saving refiner segments design," in Proceedings - PulPaper 2004 Conferences, Energy and Carbon Management, Helsinki, 2004, pp. 111-118, cited By (since 1996) 1; Conference of Proceedings - PulPaper 2004 Conferences, Energy and Carbon Management; Conference Date: 1 June 2004 through 3 June 2004; Conference Code: 64313.
[5] O. Johansson and J. Richardson, "The effect of refining zone temperature on refining efficiency and pulp quality," in Proceedings of International Mechanical Pulping Conference, Oslo, Norway, 2005, p. 50-58.

[6] A. Karlström, "Method for guiding the beating in a refiner and arrangement for performing the method," Patent, Feb., 2000, CIB: D21D1/20; D21D1/30; (IPC1 ECLA: D21D1/20; D21D1/30.

[7] A. Karlström et al., "Refining models for control purposes." Nordic Pulp and Paper Research Journal, 2008, vol. 23:1, s. 129-138.

[8] K. B. Miles and W. May, "Flow of pulp in chip refiners," Transactions of the Technical Section - Canadian Pulp and Paper Association, vol. 16, no. 2, pp. 63-72, 1990.

[9] K. Mosbye et al., "Use of refining zone temperature measurements for refiner control," in 2001 International Mechanical Pulping Conference, 2001, p. 481-488.

[10] C. Nordling and J. Österman, Physics handbook : elementary constants and units, tables, formulae and diagrams, and mathematical formulae. Lund, Sweden; Goch, Western Germany; Bromley, Kent, England: Studentlitteratur ; Bratt Institut für Neues Lernen ; Chartwell-Bratt, 1987.

[11] X. Qian, "Modelling and dynamic simulation of CTMP plant," PhD thesis, University of British Columbia, Vancouver, 1996.

[12] S. Rönnbäck, "Lönsamt att satsa på reglerteknik," Nordisk Papper \& Massa, vol. 40, no. 3-4, pp. 40-41, 2004.

[13] D. Sikter et al., "Using the refining zone temperature profile for quality control," in 2007 International Mechanical Pulping Conference, Minneapolis, MI, USA, 2007, p. 7-9.

[14] D. Sikter et al., "Economic perspectives on quality control in TMP refining processes," Nordic Pulp and Paper Research Journal, vol. 23 , no. 03, pp. 305-314, Sep. 2008.

[15] Terrence S Fox, "PULP TRANSPORT IN THE DISK REFINER," Institute of Paper Chemistry, Progress Report, May 1979.

[16] The Swedish Forest Industries, "Facts and figures 2010," 2010.

[17] W. Wagner et al., "The IAPWS industrial formulation 1997 for the thermodynamic properties of water and steam," Journal of Engineering for Gas Turbines and Power, vol. 122, no. 1, pp. 150-180, 2000. 